\title{
Solar based Automatic Speed Control of Vehicles in Sensitive Zones
}

\author{
Janav S, Monisha S M, Pavan Kumar M G \\ B. Tech Student: \\ Dept. of Information Science and Engineering \\ New Horizon College Of Engineering \\ Bangalore, India
}

\begin{abstract}
This project aims to automatically control the speed of any vehicles in sensitive zones such as in speed limited areas,schools etc. Nowadays in a fast moving world people are not having self-control. Such people are driving vehicles in a high speed. so the police are not able to monitor all those things. This paper provides a way for how to control the speed without harming others. Driver does not control the speed in such places; controls are taken automatically by the use of electronic system. In this project we are using RF for indicating the speed limit areas it is placed front and back of the sensitive zones. RF receiver is placed inside the vehicle. Speed is controlled by the help of speedometer in the vehicle. The controller compares the speed, if it exceeds the limited speed the controller alerts the driver and controls taken automatically. If they does not respond to the message by the controller then the speed is automatically controlled.
\end{abstract}

Keywords - RF transmitter and receiver, Controller unit, Solar pannel, DC Motor, LCD.

\section{INTRODUCTION}

TAt present accidents are mostly occurs due to rash driving by the drivers and over speed on roads People do not bother about human lives. The accidents rates are increasing year to year by more vehicles on ground. The government has taken many steps to prevent this kind of things but it's not enough. Most of the manufactures has developed a laser-based control system but its cost is too high. But it is again a difficulty when human crosses the road it cannot detect properly so we tried to develop a system to control these things in a simple manner. At first, we have an idea to use laser diodes but it was costly so we go for IR module again there is a draw back in using this it works under line of sight so finally decided to use RF.

$\mathrm{RF}$ transmitter is placed in the road zone areas and receiver is placed in the vehicle. Then it transfers the information to the controller. The current speed will be monitored by the separate module or by the use of ultrasonic sensor that also sends information to the controller. The controller compares both the speed and the driver does not decreases the speed the control transfers automatically but the driver again operate it manually and exceeds the limited speed means the information transferred to the nearest police station. The

\author{
Prof. L Srinivasan \\ Assistant Professor: \\ Dept. of Information Science and Engineering \\ New Horizon College Of Engineering \\ Bangalore, India
}

information contains the current speed and registration number of the vehicle. The controller transmits the information with the help of GSM module. Then the penalty amount is paid by the owner.

Here we propose a dynamic model where the system controls the vehicle according to the data frame that is transmitted by the RF transmitter fixed to the nearby road signs. The data frame is received by the microcontroller in point-of-sale and access control. In this application the ARM 7 controller is the heart of operations of Electronic Controller and Display Unit. It consists of 2 ports for interfacing with various devices and has $40 \mathrm{~kb}$ of RAM and $512 \mathrm{~kb}$ of Flash memory and high-speed performance at a speed of $60 \mathrm{MHz}$.

\section{COMPONENTS OF ELECRICAL PANEL}

\section{a. Microcontroller}

A microcontroller is an electronic component, it takes in electrical signals, and makes decisions based on those signals then it reacts by giving out electrical signals. features a high level of integration and minimal power consumption. Due to their low power consumption and tiny size, LPC 2148 are ideal for application where miniaturization is a requirement, such as point-of-scale and access control. In this application this controller is the heart of operations of Electronic Controller and Display Unit.

\section{b. RFID Reader}

RFID reader connected to a wire antenna which demodulates the Manchester RF 32/64-bit signal and decodes it automatically. We carry RFID reader modules for every application include for long distance reading. The data retrieved from the transponder is ready to be processed inside the device or to be sent over 12C/UART/SPI. The user can control chip/module with an external device such as microcontroller.

\section{c. RF Tags}

RFID tags are a type of tracking system that uses small barcodes in order to identify items, RFID tags utilize radio frequency technology. These radio waves transmit data from the tag to a reader, which then transmits the information to an RFID computer program. 
$\mathrm{RF}$ identification is the wireless non-contact use of radiofrequency electromagnetic fields to transfer data, for identifying and tracking tags attached to objects. A Passive Clamp shell type tag is used for transmitting the road-sign which consists of memory chip and antenna.

\section{d. DC Motor}

A DC Motor is a rotary electrical motor that converts direct current electrical energy into mechanical energy. Nearly all types of DC Motors have some internal mechanism. DC Motor is a mechanically commuted electric motor powered from direct current (DC). DC motors can operate directly from batteries which are rechargeable, providing the motive power for the first electric vehicles.

\section{e. 2Relay Modules}

Electromechanical relays are devices that join or break circuit by physically moving electrical contacts into contact with each other.

\section{f. LCD Module}

The Liquid Crystal library allows you to control LCD displays that are compatible with the Hitachi HD44780 driver. There are many of them out there, and you can usually tell them by the 16-pin interface. The LCDs have a parallel interface, meaning that the microcontroller has to manipulate several interface pins at once to control the display

The process of controlling the display involves putting the data that form the image of what you want to display into the registers, then putting instructions in the instruction register. The Liquid Crystal Library simplifies this for you.

\section{g. Solar Panel}

Solar energy is an unlimited source of energy. This project has been designed keeping this in view to make the harnessing of solar energy more efficient.

Sun Tracking Solar Panel Using Arduino project is based on Arduino controller board which controls the various activities of the project. A Solar Panel is used to harness solar energy. A Solar Panel is attached to a motor, this motor is electrically connected to the controller board. The system periodically checks the availability of solar energy from one horizon to another horizon periodically. In this way maximum power that can be harnessed with the solar panel is harnessed by the system.

\section{WORKING}

When the vehicle enters in the normal area the speed does not decrease and it goes normally and no action will be performed. when the vehicle is entering into the restricted areas that means it enters into the speed limiting. Whenever it enters the restricted areas, transmitter module just sends an information that contains how much speed a vehicle should go inside the speed limited area then the signal or information is received by the receiver and the signal acquired from the speed meter is also given to the controller.the signal is basically analog in nature that will be converted into digital so only the micro controller will be able to process the signal.

The signal from the transmitter and the speed meter is compared by the controller. In this there are two case: first, If the current speed is less than the transmitted speed the vehicle goes normally no action is needed. second, the information from the speed meter is greater than the transmitted speed by the transmitter module the controller waits for few second whether the driver reduces the speed to the below value if the driver does not reduce the speed then it automatically takes the control and reduces the speed according to it. At the same time the information is transmitted to the nearest police station, the information contains the vehicle number and the time, the time denotes that at which time the vehicle crossed that area then the fine or penalty amount is collected by the nearest tollgate or the check post. After that at the end of the speed limit area there is another transmitter that contains a stop information about the control releases by the controller to the driver. This speed control system assures that the number of accidents near the school and another specific zones to reach its minimum speed. this system requires very low cost, durable, low power, and gives maximum safety to the public and simple design to implement in the specific areas. This system also works on bad weather days. This system will protect the public from the rash drivers, alcoholics, and the drivers who lost their minds while driving. By implementing this system, we can give a safe environment to the public

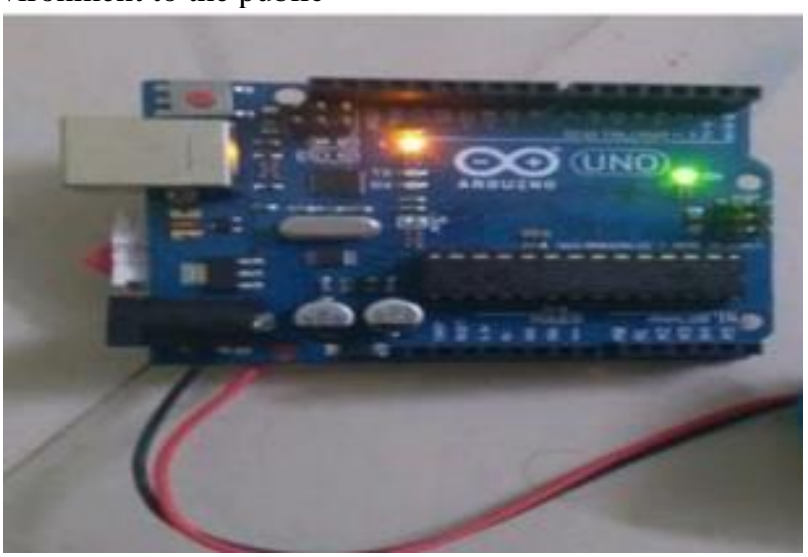

Fig.3.1. Transmitter circuit

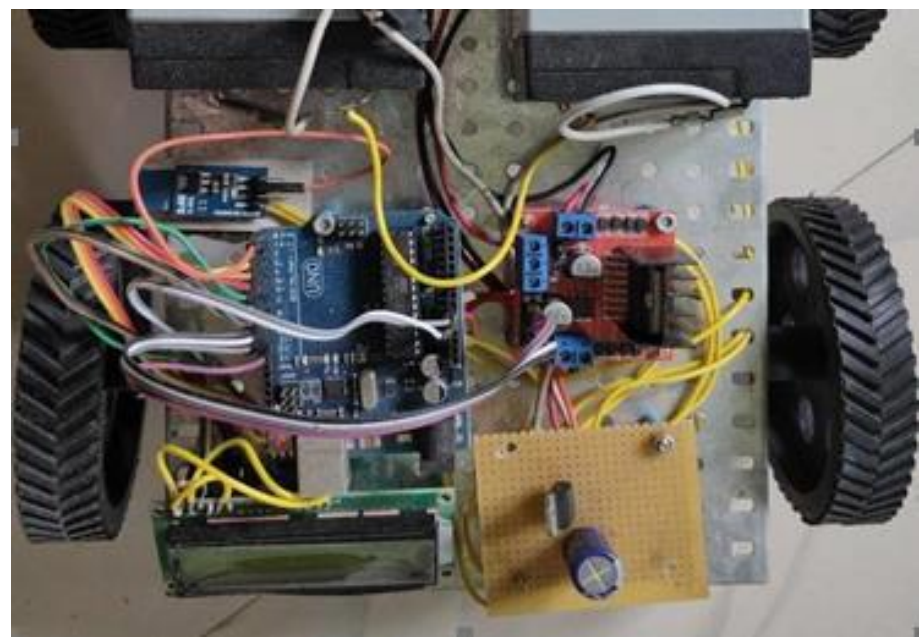

Fig.3.2. Receiver circuit

If the speed of the vehicle is less than the predefined speed that is programmed in the microcontroller than no action will be taken. If the speed of the vehicle is greater than the predefined 
speed then the microcontroller controls the speed of the vehicle by sending a signal to the motor driver in it and the motor driver reduces the speed of the electric motor.

\section{V.CONCLUSION}

This study shows the role of reducing vehicle speed automatically and its contributions to the safety of public and road users. The vehicle speed control system contributes a lot in minimizing the accident rate that occurs due to the negligence of the driver to disobeying roadside signboards in sensitive zones. Though the VSC system in a vehicle is effective, they help much in terms of improving safety, keeping both the passenger safety and the pedestrians on the roads. Considering the automatic VSC system is incorporate in school zones or hospital zones which allows the vehicle to act independently to slow down the vehicle when the vehicle comes at a higher speed which minimizes the accidents due to negligence of the drivers actively and in a way more effectively. Hence it is concluded from the above study that the uses of Solar based Automatic vehicle speed control systems in sensitive zones minimize unwanted accidents to a great extent compared to the normal behavior.

\section{VI.REFERENCES}

[1] Rooikat M. National statistics of road traffic accident in India. [2013].

[2] Robert Bosch GmbH, Driver assistance systems.

[3] Sridhar Ayer, RFID: Technology and Applications.

[4] Sanjay Sharma, Analog and digital communication, fifth revised edition (June 2010).

[5] Tom Igor, Getting Started with RFID: Identify Objects in the Physical World.

[6] Jeff Kroomen, ECUs and Engine Calibration, Performance Electronics, Ltd

[7] Akash Batra, et.al., Automatic Car Speed Control with Wireless RF Control, ISSN: 2277-9655, et al., 7(4): April 2018, pp.592-597.

[8] R. Ashok Kumar et.al., A Beacon Based Automatic Vehicle Speed Control System for Restricted Zone, Volume 7, Issue X, October 2018, pp.1152-1159.

[9] Shankhavi K B, et.al., Vehicle Speed Control using RF Communication, Volume 13, Issue 1, May 2016, ISSN: 2349 - 9303.

[10] R. Deepa. Design of Vehicle Speed Control System Using Wireless Instrument Cluster, Volume 4, Issue 1, January 2015.

[11] Sunil R Kewate, et.al., Automatic speed control system by the color sensor for automobiles - An innovative modelbased approach, ISSN 2250-3234, volume 4, number 2 (2014), pp. 223-230.

[12] Dr.K.S. Tamilselvan, et.al., Android Based Vehicle Speed Control System in Critical Zone Using GPS Technology, volume 7, issue 6, June 2018, pp.639-644.

[13] K. Govindaraju, et.al., Embedded Based Vehicle Speed Control System Using Wireless Technology, Volume 2, Issue 8, August 2014, pp.1841-1844.

[14] Gopal P. Gawande, et.al., Review of Speed Control and Automatic Braking System, Volume 3, Issue 2, February 2014, pp.474-476. 